\title{
ANTIMICROBIAL SUSCEPTIBILITY OF SALMONELLA ENTERICA SEROVARS IN A TERTIARY CARE HOSPITAL IN KOSHI REGION (NORTHERN BIHAR), INDIA
}

\author{
Syed Khursheed Ejaz ${ }^{1}$, Chandan Kumar Poddar², Maheshwar Narayan Singh 3 , Abdul Rahman Hasan', Jagarnath Prasad5, \\ Randhir Kumar6, Namita Bhowmick7, Ram Nagina Sinha8 \\ ${ }^{1}$ Assistant Professor, Department of Biochemistry, Narayan Medical College \& Hospital, Sasaram. \\ ${ }^{2}$ Research Scholar, Department of Microbiology, Indira Gandhi Institute of Medical Sciences, Patna. \\ 3 Professor, Department of Microbiology, Anugrah Narayan Magadh Medical College, Gaya. \\ ${ }^{4}$ Assistant Professor, Department of Physiology, Lord Buddha Koshi Medical College, Saharsa. \\ 5 Professor, Department of Anaesthesiology, Lord Buddha Koshi Medical College, Saharsa. \\ ${ }^{6}$ Senior Resident, Department of Microbiology, Indira Gandhi Institute of Medical Sciences, Patna. \\ ${ }^{7}$ Microbiologist, Department of Microbiology, National Test House, Salt Lake, Kolkata. \\ ${ }^{8}$ Associate Professor, Department of Surgery, Lord Buddha Koshi Medical College, Saharsa
}

\begin{abstract}
BACKGROUND

Typhoid fever continues to remain a major public health problem, especially in regions such as Koshi Region due to poor sanitation and personal hygiene. Koshi Region is often prone to enteric fever outbreaks and is an endemic region of typhoid fever. Salmonella enterica serovars Typhi and Paratyphi are predominantly known to cause enteric fever. Multidrug resistance in S. Typhi and S. Paratyphi has emerged as a cause of concern. This study was done to evaluate status in antimicrobial susceptibility patterns of Salmonella enterica serovar Typhi (S. Typhi) and S. paratyphi obtained from blood culture in our tertiary care hospital in Northern Bihar, India.
\end{abstract}

\section{MATERIALS AND METHODS}

A total of 69 isolates of Salmonella Typhi and Salmonella Paratyphi A collected from different clinical isolates were tested for antimicrobial susceptibility according to the CLSI guidelines. Blood isolates of Salmonella species over a two year period between December 2013 and December 2015 were studied. A total of 69 isolates of Salmonella species were tested for antimicrobial susceptibility by Kirby-Bauer disc diffusion method.

\section{RESULTS}

Bacterial cultures included 69 S. Typhi isolates from blood cultures of patients from suspected typhoid fever who attended the outpatient clinics or were admitted in the hospitals during December 2013-December 2015. 39 (56.52\%) were S. Typhi and 30 (43.47\%) were S. Paratyphi A. Of these isolates, 60 (86.95\%) were sensitive to ciprofloxacin (MIC $<0.25 \mathrm{mg} / \mathrm{mL}), 63(91.30 \%)$ were nalidixic acid resistant. Of the 63 nalidixic acid resistant isolates, 60 (95.23\%) were susceptible to ciprofloxacin (MIC $<0.25$ $\mathrm{mg} / \mathrm{mL}$ ). All 69 isolates were sensitive to co-trimoxazole, gentamycin and ceftriaxone, 60 isolated (86.95\%) were sensitive to amoxicillin and 63 (91.30\%) were sensitive to chloramphenicol.

\section{CONCLUSION}

Nalidixic acid resistance screening is not a reliable surrogate indicator of ciprofloxacin resistance. Ciprofloxacin MIC should be routinely done. The isolates showed a high degree of susceptibility to ampicillin, co-trimoxazole and chloramphenicol. Thus, antibiotics like amoxicillin, co-trimoxazole, and third generation Cephalosporins (Cefotaxime) may once again be useful for the management of enteric fever in our tertiary care hospital in Northern Bihar, India.

\section{KEYWORDS}

Typhoid, Multidrug Resistance, Salmonella Typhi and Salmonella Paratyphi A.

HOW TO CITE THIS ARTICLE: Ejaz SK, Poddar CK, Singh MN, et al. Antimicrobial susceptibility of salmonella enterica serovars in a tertiary care hospital in Koshi region (Northern Bihar), India. J. Evolution Med. Dent. Sci. 2017;6(22):1808-1811, DOI: $10.14260 /$ Jemds/2017/397

\section{BACKGROUND}

Enteric fever is a global public health problem. Almost $80 \%$ of the cases and deaths are in Asia and the rest occur mostly in Africa and Latin America.[1] It is estimated that there are 22

Financial or Other, Competing Interest: None.

Submission 07-02-2017, Peer Review 02-03-2017,

Acceptance 09-03-2017, Published 16-03-2017.

Corresponding Author:

Chandan Kumar Poddar,

M. Sc., Medical Microbiology,

BU Jhansi, Ph. D, (Students) IGIMS, Patna.

E-mail: chandan_microbiology@yahoo.co.in

DOI: $10.14260 /$ jemds $/ 2017 / 397$ million new cases of enteric fever annually, with 200,000 deaths. [2] Regions with the highest incidence of enteric fever (>100 cases per 100,000 persons per year) are South Central Asia and Southeast Asia. Regions of moderate incidence (10-100 cases per 100,000 persons per year) include the rest of Asia, Africa, Latin America and the Caribbean and Oceania, except for Australia and New Zealand. In Delhi, India, the incidence of enteric fever is 9.8 cases per 1,000 personyears. ${ }^{[3]}$ Salmonella enterica serovar Typhi and Paratyphi A are the predominant types of aetiological agents responsible for enteric fever in India, particularly during summer.[4] The emergence of antimicrobial resistance, especially the multidrug resistance to ampicillin, chloramphenicol and co- 
trimoxazole, has further complicated the treatment and management of enteric fever. $[4,5]$

In India, antibiotic resistance among S. Typhi has been reported since 1960, and the first outbreak of multidrug resistant S. Typhi (MDRST) was reported in Calicut.[6] Since then MDRST has appeared throughout the world, especially in South America, the Indian subcontinent, Africa and Southeast Asia.[5] The incidence of multidrug resistant (MDR) $\mathrm{S}$. Typhi has been reported to be as high as $60 \%$ but then declined in Pune (1999), Nagpur (2001), Delhi (2004) and Calcutta (2000).[7-10] However, resurgence of resistant strains in Ludhiana in 2002 is of concern.[11] A study of imported strains that was based in the United States ${ }^{[12]}$ noted an increase in the number of MDR and nalidixic acid resistant $S$. Typhi globally (NARST), although all isolates remained sensitive to ciprofloxacin and ceftriaxone. In Bangladesh there has been a reported decrease in MDR isolates with no corresponding increase in sensitive strains.[13] For ciprofloxacin there has been an increase in MIC strains imported into the United Kingdom,[14] Bangladesh,[15] and India. $[16,17,18]$

Multidrug resistant (MDR) strains (resistant to chloramphenicol, ampicillin and co-trimoxazole) of Salmonella enterica have emerged worldwide in the last two decades. Isolates of $\mathrm{S}$. enterica with reduced susceptibility to fluoroquinolones have now appeared in the Indian subcontinent and other regions.[19,20] However, in India the degree of resistance to commonly used antibiotics such as chloramphenicol, ampicillin and co-trimoxazole in the era of quinolone resistance is not clear.[21,22] The present study was undertaken to document the change in the antibiotic susceptibility of S. enterica serovar Typhi and S. Paratyphi isolates obtained from blood culture during 2013-2015 in a tertiary care hospital in Northern Bihar, India.

\section{MATERIALS AND METHODS}

\section{Study Design}

All S. enterica isolates obtained from blood cultures of clinically suspected cases of enteric fever seen in Lord Buddha Hospital (Central Hospital) and Associated Hospital, Lord Buddha Koshi Medical College, a tertiary care center in Koshi Region (Northern Bihar), from December 2013 to December 2015 were included in the study. The study protocol was approved by the hospital ethics committee.

\section{Criteria for selection of patients-}

Patients included in this study had fever for less than 3 days, had no anti-typhoid treatment, or any condition suggestive of typhoid (Soft enlarged spleen, headache, and abdominal discomfort with diarrhoea). Members of both sexes representing all ages will included.

Bacterial Cultures included 69 S. Typhi isolates from blood cultures of patients suffering from suspected typhoid fever who attended the outpatient clinics or were admitted in the hospitals during December 2013-December 2015 in a tertiary care hospital in Koshi Region (Northern Bihar), India. A total of 1,000 blood samples of Bile salt broth (broth culture),[23] and streptokinase broth (clot culture)[24] blood samples were used for enrichment as well as Selenite F-broth for environmental samples. The enriched samples after visible turbidity were streaked on MacConkey, XLD and
Wilson Blair media. All isolates were identified by biochemical test.

\section{Antimicrobial Susceptibility Testing}

Antimicrobial susceptibility patterns were determined using commercial antimicrobial disks (HiMedia, Mumbai): Chloramphenicol (30 $\mu \mathrm{g})$, nalidixic acid (30 $\mu \mathrm{g})$, ampicillin $(10 \mu \mathrm{g})$, azithromycin $(15 \mu \mathrm{g})$, co-trimoxazole $(1.25 / 23.75$ $\mu \mathrm{g})$, ciprofloxacin $(5 \mu \mathrm{g})$, and ceftriaxone $(30 \mu \mathrm{g})$. Antimicrobial susceptibility testing was performed in accordance with the Clinical and Laboratory Standards Institute (CLSI) guidelines by Kirby-Bauer disc diffusion method.[25]

MICs of isolates resistant to chloramphenicol, ampicillin and nalidixic acid were determined by agar dilution test using purified antibiotic powders (HiMedia Laboratories, Mumbai). MIC of ciprofloxacin were determined for only 20 randomly selected nalidixic acid resistant isolates. Isolates resistant to ampicillin, chloramphenicol and cotrimoxazole were termed MDR.

\section{RESULTS}

Of the total 69 isolates studied, 39 (56.52\%) were S. Typhi and $30(43.47 \%)$ were S. Paratyphi A. Of these isolates, 60 (86.95\%) were sensitive to ciprofloxacin (MIC $<0.25 \mathrm{mg} / \mathrm{mL}$ ), $63(91.30 \%)$ were nalidixic acid resistant. Of the 63 nalidixic acid resistant isolates, $60(95.23 \%)$ were susceptible to ciprofloxacin (MIC $<0.25 \mathrm{mg} / \mathrm{mL}$ ). All 69 isolates were sensitive to co-trimoxazole, gentamycin and ceftriaxone, 60 isolated $(86.95 \%)$ were sensitive to amoxicillin and $63(91.30 \%)$ were sensitive to chloramphenicol (see Table below).

Enteric fever is a major public health problem in India. Various studies document S. Typhi as the commonest serovar isolated over the years,[26] and our study also showed 56.52 percent isolates of serovar Typhi while 43.47 percent were serovar Paratyphi A.[27]

\begin{tabular}{|c|c|c|}
\hline Antibiotics Screened & No. of Isolates & Sensitive Isolates \\
\hline Nalidixic Acid $(30 \mu \mathrm{g})$ & 69 & $6(08.69 \%)$ \\
\hline Amoxicillin $(10 \mu \mathrm{g})$ & 69 & $60(86.95 \%)$ \\
\hline Chloramphenicol $(30 \mu \mathrm{g})$ & 69 & $63(91.30 \%)$ \\
\hline $\begin{array}{c}\text { Co-trimoxazole } \\
(1.25 / 23.75 \mu \mathrm{g})\end{array}$ & 69 & $69(100 \%)$ \\
\hline Ciprofloxacin $(5 \mu \mathrm{g})$, & 69 & $60(86.95 \%)$ \\
\hline Ceftriaxone $(30 \mu \mathrm{g})$ & 69 & $69(100 \%)$ \\
\hline \multicolumn{2}{|c|}{ MDR* $^{*}$ Sensitivity Rates to Various Antibiotics in } \\
A Tertiary Care Hospital in Northern Bihar, India \\
\hline
\end{tabular}

*Defined as resistance to amoxicillin, chloramphenicol and co-trimoxazole

\section{DISCUSSION}

Due to a combination of factors including poor sanitation and health care infrastructure, typhoid fever remains a major public health problem in most resource-poor countries such as India. This is the first report of S. Typhi antimicrobial susceptibility from Northern Bihar, India. Enteric fever is a major infectious disease occurring at high fluctuating incidences in tertiary care center in Koshi Region (Northern Bihar), Lord Buddha Koshi Medical College (Northern Bihar). 
In the last decade, there have been some reports of ciprofloxacin resistance in Salmonella.[28] It is believed that nalidixic acid resistance is a surrogate marker for ciprofloxacin resistance, as clinical failures have been documented in cases where ciprofloxacin has been used (based on susceptibility) for nalidixic acid resistant strains.[29] In our study, 13.04 percent of isolates displayed reduced susceptibility to ciprofloxacin (MIC $>0.5 \mu \mathrm{g} / \mathrm{mL}$ ). However, as many as 91.30 percent of nalidixic acid resistant isolates were ciprofloxacin sensitive by MIC testing. KirbyBauer disc diffusion assay using currently recommended breakpoints to ciprofloxacin may not be a reliable method; Etest should be the preferred method of choice to determine ciprofloxacin MIC.

Since its introduction in 1948, chloramphenicol has been the treatment of choice for typhoid fever and remains the standard against which newer antimicrobials are compared. Treatment with chloramphenicol reduces mortality due to typhoid fever from about 20 to 1 percent and the duration of fever from 14-28 days to 3-5 days.[30] However, chloramphenicol therapy has been associated with the emergence of resistance to chloramphenicol, a high relapse rate, bone marrow toxicity and high mortality rates in a recent study reported from the developing world.[31] Ampicillin and co-trimoxazole could be effective alternative drugs. In our study, Salmonella spp. remained sensitive to chloramphenicol, amoxicillin, ceftriaxone and co-trimoxazole (91.30, 86.96, $100 \& 100 \%$, respectively) over the two-year study period as reported earlier.[32,33] These drugs may be preferred for treatment of enteric fever in Northern Bihar, India.

The data presented in our study highlights that MDR, although small, exists in this region. The presence of MDR (i.e. resistance to ampicillin, chloramphenicol and cotrimoxazole) was $8.69 \%$ for $2013-2015$. This finding is in accordance with recent reports from some regions where the incidence of MDR S. Typhi isolates appeared to have decreased.[32-34] The low frequency of MDR S. Typhi isolated is remarkable, since these drugs could once again be used for the treatment of enteric fever.[10,34,35]

Azithromycin has done well in previous clinical studies for typhoid; however, there have been sporadic reports of azithromycin resistance.[36] All isolates in our study were sensitive to ceftriaxone in contrast to some studies that reported resistance to ceftriaxone.[37,38]

A limitation of our study was that clinical outcomes were not analysed. Quinolones may remain effective despite in vitro resistance and ceftriaxone may be associated with prolonged time to fever resolution despite in vitro sensitivity.[39]

\section{CONCLUSION}

The main approaches for combatting typhoid fever today is the control of the dissemination of causative agent. To this end, investigation of S. typhi is of major importance.

Nalidixic acid resistance screening is not a reliable surrogate indicator of ciprofloxacin resistance. Ciprofloxacin MIC should to be routinely done. Azithromycin resistance appears to be emerging. However, isolates showed a high degree of susceptibility to ampicillin, co-trimoxazole and chloramphenicol. Thus, antibiotics like amoxicillin, cotrimoxazole, and third generation Cephalosporins (Cefotaxime) may once again be useful for the management of enteric fever in our tertiary care hospital in Northern Bihar, India. However, chloramphenicol, co-trimoxazole and amoxicillin have re-emerged as valuable oral options and ceftriaxone remains a viable parenteral option for treatment of typhoid in our tertiary care hospital in Northern Bihar, India.

\section{REFERENCES}

[1] The World Health Report, Report of the Director General WHO. World Health Organisation: Geneva. 1996.

[2] Crump JA, Luby SP, Mintz ED. The global burden of typhoid fever. Bull World Health Organ 2004;82(5):346-53.

[3] Sinha A, Sazawal S, Kumar R, et al. Typhoid fever in children aged less than 5 years. Lancet 1999;354(9180):734-7.

[4] Jesudason MV, John TJ. Plasmid mediated multidrug resistance in Salmonella typhi. Ind J Med Res 1992;95:66-7.

[5] Mourad AS, Metwally M, Nour Ei Deen A, et al. Multiple drug-resistant Salmonella typhi. Clin Infect Dis 1993;17:135-6.

[6] Agarwal SC. Chloramphenicol resistance of Salmonella species in India, 1956-61. Bull World Health Organ 1962;27(3):331-5.

[7] Sanghavi SK, Mane MP, Niphadkar KB. Multidrug resistance in Salmonella serotypes. Ind J Med Microbiol 1999;17(2):88-90.

[8] Chande C, Shrikhande S, Kapale S, et al. Change in antimicrobial resistance pattern of Salmonella typhi in central India. Indian J Med Res 2002;115:248-50.

[9] Saha MR, Dutta P, Niyogi SK, et al. Decreasing trend in the occurrence of Salmonella enterica serotype typhi amongst hospitalised children in Kolkata, India during 1990-2000. Indian J Med Res 2002;115:46-8.

[10] Walia M, Gaind R, Mehta R, et al. Current perspectives of enteric fever: a hospital based study from India. Ann Trop Paediatr 2005;25(3):161-74.

[11] Kumar R, Aneja KR, Roy P, et al. Evaluation of minimum inhibitory concentration of quinolones and third generation cephalosporins to Salmonella typhi isolates. Indian J Med Sci 2002;56(1):1-8.

[12] Ackers ML, Puhr ND, Tauxe RV, et al. Laboratory-based surveillance of Salmonella serotype typhi infections in the United States: antimicrobial resistance on the rise. JAMA 2000;283(20):2668-73.

[13] Rahman M, Ahmad A, Shoma S. Decline in epidemic of multidrug resistant Salmonella typhi is not associated with increased incidence of antibiotic susceptible strain in Bangladesh. Epidemiol Infect 2002;129(1):29-34.

[14] Threlfall EJ, Ward LR. Decreased susceptibility to ciprofloxacin in Salmonella enterica serotype typhi, United Kingdom. Emerg Infect Dis 2001;7(3):448-50.

[15] Asna SM, Haq JA, Rahman MM. Nalidixic acid resistant Salmonella enterica serovar typhi with decreased 
susceptibility to ciprofloxacin caused treatment failure: a report from Bangladesh. Jpn J Infect Dis 2003;56(1):32-3.

[16] Bhat KG, Suresh K. Ciprofloxacin-resistant Salmonella typhi. Natl Med J India 1999;12(2):88.

[17] Jesudason MV, Malathy B, John TJ. Trend of increasing levels of minimum inhibitory concentration of ciprofloxacin to Salmonella typhi. Indian J Med Res 1996;103:247-9.

[18] Harish BN, Madhulika U, Parija SC. Current pattern in antimicrobial susceptibility of Salmonella typhi isolates in Pondicherry. Indian J Med Res 2004;120(2):111-4.

[19] Brown JC, Shanahan PM, Jesudason MV, et al. Mutations responsible for reduced susceptibility to 4quinolones in clinical isolates of multi-resistant Salmonella typhi in India. J Antimicrob Chemother 1996;37(5):891-900.

[20] Effa EE, Bukirwa H. Azithromycin for treating uncomplicated typhoid and paratyphoid fever (enteric fever). Cochrane Database Syst Rev 2008;(4):CD006083.

[21] Menezes GA, Harish BN, Khan MA, et al. Antimicrobial resistance trends in blood culture positive Salmonella typhi isolates from Pondicherry, India, 2005-2009. Clin Microbiol Infect 2012;18(3):239-45.

[22] Harish BN, Menezes GA. Preserving efficacy of chloramphenicol against typhoid fever in a tertiary care hospital, India. Reg Health Forum 2011;15(1):926.

[23] Watson KC. Clot culture in typhoid fever. J Clin Pathol 1954;7(4):305-7.

[24] Watson KC. Laboratory and clinical investigation of recovery of Salmonella typhi from blood. J Clin Microbiol 1978;7(2):122-6.

[25] Clinical and Laboratory Standards Institute (CLSI) Performance standards for antimicrobial disk susceptibility tests. Approved standard. 9th edn. CLSI Document M2-A9. Wayne, PA: CLSI; 2006.

[26] Manchanda V, Bhalla P, Sethi M, et al. Treatment of enteric fever in children on the basis of current trends of antimicrobial susceptibility of Salmonella enterica serovar typhi and paratyphi A. Indian J Med Microbiol 2006;24(2):101-6.
[27] Chandel DS, Chaudhry R, Dhawan B, et al. Drugresistant Salmonella enterica serotype paratyphi A in India. Emerg Infect Dis 2000;6(4):420-1.

[28] Nath G, Tikoo A, Manocha H, et al. Drug resistance in Salmonella typhi in north India with special reference to ciprofloxacin. J Antimicrobials Chemother 2003;46:145-53.

[29] Mandal S, Mandal MD, Pal VK. Nalidixic acid resistance predicting reduced ciprofloxacin susceptibility of Salmonella enterica serovar typhi. Asian Pacific J Trop Dis 2012;2(Suppl 2):S585-7.

[30] Mirza SH, Beeching NJ, Hart CA. Multi-drug resistant typhoid: a global problem. J Med Microbiol 1996;44(5):317-9.

[31] Nagshetty K, Channappa ST, Gaddad SM. Antimicrobial susceptibility of Salmonella typhi in India. J Infect Dev Ctries 2010;4(2):70-3.

[32] Parry CM. The treatment of multidrug-resistant and nalidixic acid-resistant typhoid fever in Vietnam. Trans R Soc Trop Med Hyg 2004;98(7):413-22.

[33] Tankhiwale SS, Agrawal G, Jalgaonkar SV. A preliminary report on current antibiogram of Salmonella enterica serotype typhi in Nagpur. Indian J Med Microbiol 2003;21(4):292.

[34] Rodrigues C, Shenai S, Mehta A. Enteric fever in Mumbai, India: the good news and the bad news. Clin Infect Dis 2003;36(4):535.

[35] Mandal S, Mandal MD, Kumar NP. Reduced minimum inhibitory concentration of chloramphenicol for Salmonella enterica serovar typhi. Indian J Med Sci 2004;58(1):16-23.

[36] Pfeifer Y, Matten J, Rabsch W. Salmonella enterica serovar typhi with CTX-M beta-lactamase, Germany. Emerg Infect Dis 2009;15(9):1533-5.

[37] Molloy A, Nair S, Cooke FJ, et al. First report of Salmonella enterica serotype paratyphi A azithromycin resistance leading to treatment failure. J Clin Microbiol 2010;48(12):4655-7.

[38] Gokul BN, Menezes GA, Harish BN. Acc-1 $\beta$-lactamaseproducing Salmonella enterica serovar Typhi, India. Emerg Infect Dis 2010;16(7):1170-1.

[39] Jog S, Soman R, Singhal T, et al. Enteric fever in Mumbai-clinical profile, sensitivity patterns and response to antimicrobials. J Assoc Physicians India 2008;56:237-40. 